\title{
EL AIMIRANTE DON CRISTÓBAL COLÓN EN LA CORTE DE LOS REYES CATÓXICOS EN 1497. ESTANCIAS EN VALLADOLID, BURGOS Y MEDINA DEL CAMPO. EL "MEMORIAL DEL ORO QUE SE HA VENDIDO EN CASTILA"
}

por

\section{ANTONIO RUMEU DE ARMAS}

Academia de la Historia.

RESUMEN: El estudio aporta nuevos datos sobre el itinerario del Almirante en el reino de Castilla entre julio de 1496 y marzo de 1497, desde su llegada a Cádiz procedente del Nuevo Mundo basta su encuentro con los Reyes Católicos en Burgos. Está basado en la información contenida en una manuscrito autógrafo de Colón, titulado "Memorial del oro gastado en Castilla», donde da cuenta de los gastos realizados durante este viaje, que se incorpora en apéndice.

Palabras clave: Edad Moderna. Castilla. Descubrimientos. Monarquía. Metales preciosos.

ABSTRACT: This paper is giving more details on the Admiral's itinerary in the Kingdom of Castilla between July 1496, the date of bis arrival to Cadiz and March 1497, whem he met the Catholic Kings in Burgos. The main bistorical source is a manuscript written by Colon, with the tiile "Memorandum of the gold spent in Castillan, where be took an account of the expenses be had during bis treap. This document is included as appendix.

KEY WORDS: Early Modern History. Castilla. Discoveries. Monarchy. Treasures.

1. EL ALMMRANTE DISPONE VIAJE A LA CORTE. EL SEQUTTO. ALONSO SÁNCHEZ DE CARVAJAL Y JUAN ANTONIO COLOMBO

El Memorial del oro vendido en Castilla es un manuscrito autógrafo de Cristóbal Colón, conservado en el importante archivo de la casa ducal de Alba, en el palacio de Liria de Madrid ' ${ }^{1}$. Su simple lectura lleva el ánimo a la conclusión de

1 Se conserva en dichos fondos; pero no se hace constar la signatura.

Hi.pania, LIX/3, nim. 203 (1999) 857-870 
que se trata del itinerario de un viaje emprendido por el almirante dentro del ámbito geográfico de Castilla la Vieja, en compañia de dos colaboradotes conocidos con los nombres de Alonso Sánchez de Carvajal y Juan Antonio Colombo. Parten de Sevilla, hacen escala en Valladolid y Arcos y se instalan en Burgos para una larga estadía.

En este viaje el almirante llevaba consigo una buena bolsa de oro en polvo coronario, que iba vendiendo al peso en las paradas del camino para atender a los gastos inherentes al transporte y hospedaje de su propia persona y séquito. La minuciosa cuenta, redactada de puño y letra, nos servirá de guía ${ }^{2}$.

Diversos autores se han devanado los sesos para datar el documento. De más temprano a más tardío, J. Gil y C. Varela optan por el año 1500 ○ 1501; De Lollis por 1502 o 1503, y Thacher por 1504 o principios de $1505^{3}$.

Desde nuestro punto de vista el curioso documento hay que datarlo en 1496-1497 por la doble motivación que pasamos a desarrollar:

1. A lo largo de la permanencia de Colón al servicio de la Corona de Castilla, los soberanos, es decir, los Reyes Católicos se establecen en Burgos durante el largo periodo que se extiende desde el 22 de septiembre de 1496 hasta el 9 de mayo de 1497. El año anterior, 1495, se alojan por espacio de un bimestre, pero en este breve periodo el almirante se hallaba gobernando la isla Española ${ }^{4}$. Ni antes ni después de estas fechas buscaron cobijo en la caput Castelle.

2. La presencia en Burgos de Alonso Sánchez de Carvajal, en 1497, está certificada por la pluma del propio almirante. En una carta escrita en Sevilla al obispo de Badajoz Juan de Fonseca, y que debe datarse en los dias postreros de 1497, se expresa así: «Al tiempo que yo salí de la corte (Medina del Campo, verano del año expresado) estando el Rey e la Reina...juntos, les dixe que pues no se podía remediar en la paga de alguna presonas de bien, que yo traia conmigo,... que se debe pagar a Alfonso Sánchez de Carvajal todo el dinero que se le debe de su servicio...» ${ }^{5}$.

En el documento que nos viene ocupando tiene un lugar destacado Juan Antonio Colombo, upersonajillo" que se incorpora al séquito del almirante, en Burgos, en febrero de 1497.

Si dos entrañables colaboradores de Cristóbal Colón han dejado en el $\mathrm{Me}$ morial del oro la huella de su nombre, parece justo dedicarles el honor de una breve semblanza biográfica.

2 Duguesa de Berwick y de Alba: Autógrafos de Cristóbal Colón, Madrid 1892, pp. 21-22.

Reproduce el documento en este trabajo en facsimil y se transcribe íntegro en Apéndice.

3 VARela, C.: Documentos Colombinos de la Casa de Alba, Madrid-Sevilla 1987, p. 27. Lollis, C.: Raccolta Colombiana, tomo I, vol. I, p. 320. THACHER, J. B. : Christopber Columbus. His life, his Works, his Remains. Nueva York 1967, tomo III pp. 378-379.

4 Rumeu de Armas, A.: Itinerario de los Reyes Católicos, Madrid 1974, pp. 229-234 y 218.

5 Real Academia de la Historia. Colection Muñoz, A/102, fols. 191v-192.

Hippania, LIX/3, núm. 203 (1999) 857-870 
El primero de los actores, Alonso Sánchez de Carvajal, era un personaje de nota en la corte de los Reyes Católicos, ejerciendo el preciado cargo de contino. Había nacido en Baeza en el seno de una ilustre familia, siendo regidor perpetuo de la misma. Cristóbal Colón se sintió identificado con su persona, con reiteradas pruebas de afecto y amistad. Participó en las expediciones segunda y tercera al Nuevo Mundo.

En la etapa intermedia fue designado miembro de la junta de gobierno de La Española mientras se verificaba la laboriosa exploración de la isla de Cuba. En 1495 regresa a la metrópoli en la escuadra de Antonio de Torres para abogar por los intereses de su patrono.

En 1496, al producirse el retorno del almirante, se integra en su séquito, visitando la corte durante las jornadas regias en Burgos y Medina del Campo, conforme se ha dicho y se insistirá más adelante.

En la tercera expedición descubridora estuvo al mando de uno de los más importantes navios. Hay que destacar la habilidad diplomática de que hizo gala para reducir al rebelde Francisco Roldán.

En 1502 embarca para La Española como tepresentante personal de Cristóbal Colón cerca del gobernador Ovando, en defensa de sus intereses económicos. Por tal razón no pudo tomar parte en el cuarto y último viaje colombino. Los abusos y despojos del gobernador le incitaron a retornat a Castilla, en 1503, para denunciar las tropelías cometidas.

En 1504 el almirante y su leal colaborador se encuentran en Sevilla en la etapa postrer de la existencia del héroe, sumido en la enfermedad y ansioso de nuevas aventuras. Durante este triste epílogo Carvajal actuó de mensajeto en la corte, en continuos viajes y angustiosa compañía.

Cuando en 1505 el almirante se dispone a llevar a cabo el último viaje cortesano, que acabará en definitivo tránsito terrenal, el leal servidor se halló presente, prestándole apoyo físico y moral ${ }^{6}$.

El segundo actor que nos interesa destacar es Juan Antonio Colombo, deudo del almirante, a cuyo servicio estuvo aunque dejando escasos datos de su permanencia en España. La primera noticia que alcanzamos tiene a Génova por escenario; en un documento de 11 de octubre de 1496 manifestó el propósito de trasladarse a España para servir al almirante "Christoforum de Colombo' ${ }^{7}$. Pocos meses después se integraba en Burgos al séquito del pariente encumbrado. La presencia en Sevilla se constata el 30 de diciembre de 1497 actuando como mayordomo de su patrono en asuntos de carácter económico ${ }^{8}$.

6 CASAs, Bartolomé de las: Historia de las Indiar, Fondo de Cultura Económica. México 1951, tomo I, pp. 348, 383 y 497: tomo $\Pi$, pp. 64, 66, 69, 75, 76, 78-79, 85, 92, 93-97, 107 y 218. BuLussteros Bererta, Antonio: Cristóbal Colón, Edición Salvat. Barcelona, tomo II, pp. 269, 526, 539, 638, 651-652, 658, 660, 664-665, 671, 673, 675, 700, 703. RuMEu de ARMAs, A.: Libro Copiador de Cristóbal Colons. Ediciones Testimonio, Madrid 1989, tomo I, pp. 268, 271-272, 283.

7 Colección documental del Descubrimiento (1470-1506); tomo II, número 341.

8 Gil, J.: «Las cuentas de Cristóbal Colón», en Anuario de Estudios Hispano Americanos, tomo XLI (año 1984), p. 426. 
El almirante integró al deudo al año siguiente, 1498, en la tercera expedición al Nuevo Mundo al mando de uno de los navíos, confiándole alguna importante comisión ${ }^{9}$. Sabemos de su continuada presencia en España y América, bien que sin detalles precisos.

Andrea, hermano de Juan Antonio se trasladó posteriormente a España. El almirante le confió el mando de un navio en 1502, en la última de las expediciones descubridoras ${ }^{10}$.

\section{EL MONASTERIO DE GUADALUPE PRIMERA ESCALA. EL CUMPLIMTENTO DE UNA PROMESA}

Ya se ha hecho reiterada mención de que nuestro objetivo se centra en estudiar las incidencias sobrevenidas a la etapa inmediata al arribo a España de Cristóbal Colón en 1496, despues de rematar su segundo viaje de asentamiento en la isla Española y exploración de Cuba. La empresa se había visto coronada por el éxito en cuanto a nuevos descubrimientos; pero la colonización se saldaba con estrepitoso fracaso.

El almirante, obsesionado por que no mermase su prestigio personal en la corte, decidió el retorno haciendo su entrada en la bahía de Cádiz el 11 de junio de 1496. En el acto escribió una misiva a los reyes protectores Fernando e Isabel, a la que respondieron éstos el 12 de julio morando en la villa de Almazán, con una expresiva letra de parabienes.

Los soberanos de Castilla, ocupados en serios menesteres — boda del heredero don Juan y contienda fronteriza contra Francia - le dan largas a su empeño: «Y pues decís que seréis acá presto, debe ser vuestra venida cuàndo os pareciere que non os dé trabajo, pues que en lo pasado habéis trabajado" ".

Cristóbal Colón va a permanecer en Sevilla hasta la última decena del mes de julio. Para los gastos del viaje da orden a su maestresala de desprenderse de la primera partida de metal precioso. En el Memorial se lee la siguiente partida:

«A XX de julio, en Sebilla, vendió Cristóbal de Torres 2 marcos, 7 onças, 4 ochavas de oro a 453 el peso del castellano».

Sabía el almirante por informes privados que la corte se preparaba para una larga jornada en Burgos, escenario previsto para la boda principesca, y a esta importante ciudad castellana dirigió sus pasos sin pausa y sin prisa.

La primera escala tuvo por escenario el monasterio de Guadalupe en cumplimiento de una promesa de visitar a la Virgen extremeña formulada 67.

9 Ballesteros: Crisóbal Colón, pp 362-363 y Rumeu de Armas: Libro Copiador, tomo II, p.

10 BALESTEROS, p. 683. Colección documental del Descubrintiento, tomo IIY, números 814 y 815.

11 Colectión documental..., tomo II, número 334

Higpania, L.IX/3, núm. 203 (1999) 857-870 
el 14 de febrero de 1493, en medio de las tempestades del tornaviaje descubri$\operatorname{dor}^{12}$.

El viernes 29 de julio se constata su presencia en el cenobio en compañía de tres servidores leales: el capitán Antonio de Torres, el alguacil mayor Pedro Hernández Coronel y el comendador Gabriel de Valera. Al séquito se había incorporado también conforme sabemos el contino Alonso Sánchez de Carvajal ${ }^{13}$.

\section{LA ESTANCIA EN VALLADOLID. EL DESCUBRIDOR COMPRA VESTIMENTA NUEVA}

La caravana se puso después en movimiento obligados los viajeros a cambiar de ruta para alcanzar el corazón de Castilla. La segunda parada de la comitiva fue Valladolid, donde procedió el contino-administrador a deshacerse de una importante cuantía de oro. Véase el pertinente texto:

«A 12 d'agosto en Valladolid, vendió Carbajal 2 marcos, 6 uncias, 4.ochavaș, 3 tomines y lo dio casi todo en ropa que se compróm

El cambio de vestimenta requiere una explicación. El almirante, por exacerbado espiritu religioso, había efectuado la travesía y el desembarco con un extraño y miserable ropaje. El cronista Andrés Bernáldez, más conocido como "el cura de Los Palacios", lo describe así (tal como lo contempló en su morada sevillana): venía evestido de unas raposas de color a ábitos de fraile de Sant Francisco de Observancia, e en la fechura poco menos que ábito, e un cordón de Sant Francisco por devoción’ ${ }^{14}$.

¿Cómo iba a presentarse en la corte un almirante de las Indias con aquellos harapos? Fue, por tanto, un sastre vallisoletano quien transfornió a Colón en vistoso personaje de honor en los inminentes esponsales del heredero.

En el camino hacia Burgos, sin que se pueda señalar el lugat, conoció nuestro protagonista la inmediata partida del puerto de Laredo de la escuadra que iba a conducit a Flandes a la infanta doña Juana para contraer matrimonio con el archiduque Felipe de Habsburgo. El inmortal nauta pidió entonces papel y

12 El Diario de la Primera Navegación. Editado por Manuel Alvar, Testimonio, Madrid 1984, pp. 101-102

13 Archivo de/Monasterio de Guadalupe. Libro $1^{\circ}$ de Bautismos, fol $1 \mathrm{v}$. El viernes 29 de julio recibieron las aguas bautismales los indios Cristóbal y Pedro "criados del señor almirante don Christóbal Colóny. Actuaron de padrinos los personajes citados. Es de advertir que Gabriel de Valera es generalmente conocido en los documentros de la época con los nombres de bachiller gallego y comendador Gallego. AcEMEl, Isidoro: «Pata la historia de Colón. Nuevos datos del Atchivo de Guadalupes, en la revista ElMonasterio de Guadalupe, números 8, 10, 12 y 13 (años 1916-1917), pp. 171-172, 218-222, 266-268 y 2-4. Ramos, Demetrio: «Las visitas de Colón a Guadalupe y el cumplimiento del voto del viaje de retornon, en la revista Guadalupe, números 674-675 (año 1985), pp. 13-32.

14 Memorias del reinado de los Reyes Católicos. Edición y estudio por Manuel Gómez Moreno y Juan de M.Carriazo, Madrid 1862, pp. 333-334.

Hispania, LIX/3, núm. 203 (1999) 857-870 
pluma y escribió una meditada misiva para la reina Isabel con recomendaciones náuticas para facilitar la travesía.

La soberana de Castilla quiso dejar patente su reconocimiento al leal servidor y pergeñó en el puerto cántabro una sustanciosa epístola escrita el 18 de agosto que llenó de contento al almirante. He aquí uno de sus párrafos: «Vi vuestra letra, e la escritura e parescer vuestro para el viaje de la archiduquesa, mi muy cara e amada hija; el cual es muy bueno, es como de ome sabio e que tiene mucha plática e experiencia en las cosas de la mar.... ${ }^{15}$. La carta regia tuvo que llegar a manos del insigne navegante durante una de sus paradas.

En la parsimoniosa caminata de Cristóbal Colón con su séquito se impuso una nueva detención en la villa de Arcos. En la escala tuvo imperiosa necesidad de vender oro, como se acredita en la partida siguiente:

«11 de setiembre, en Arcos, vendió Carbajal 6 uncias, 2 ochavas, 1 tomín, 3 granos, vendiólo a...”

\section{ENTREVISTA EN BURGOS DE LOS REYES CATÓLICOS CON CRISTÓBAL COLÓN. PRESENTES AURIFEROS}

Cristóbal Colón estaba en contacto directo con la corte por medio de correos. De esta manera así que supo que la teina Isabel dirigía los pasos hacia Burgos, aceleró la caminata, pudiendo hacer la entrada en la capital castellana a mediados de septiembre.

La soberana atravesó las calles de Burgos, entre homenajes y vítores, el día 22 del mes expresado, mientras el aragonés retrasaba el acceso al 20 de octubre, por causa de la guerra contra Francia. Ambos van a permacer en la caput Castelle hasta el 9 de mayo de $1497^{16}$.

Para atender a la subsistencia propia y del séquito el almirante tuvo que desprenderse de diversas partidas de oro entre el mes de septiembre de 1497 y el 3 de marzo de 1498. Actuaron de mediadores Alonso Sánchez de Carvajal y Juan Antonio Colombo, este últirro integrado en el séquito en los primeros días de febrero. El Memorial es parco en detalles, pero sin dejar de set sustancioso. Véanse las diversas partidas de la etapa:

«19 de setiembre, en Burgos, se pesó 4 uncias, 7 ochavas, 3 tomines.

[grano] a 7 de octubre, en Burgos, vendió Carbajal 7 uncias, 4 ochavas, 5 tomines, 3 granos a 445

25 d’otubre, en Burgos, vendió Carbajal 7 uncias, 4 ochavas, 1 tomin, 10 granos a 445

12 de noviembre, en Burgos, vendió Carbajal 1 marco, 6 uncias, 7 ochavas menos 14 granos, a 448

15 Colección documental del Descubrimiento, tomo II, número 339.

16 Rumeu de ARmas: Itinerario de los Reyes Católicos, Madrid 1974, pp. 229-234.

Hispania, LIX/3, núm 203 (1999) 857-870 
17 de diziembre, vendió en Burgos Carbajal, 7 uncias, 5 ochavas, 3 tomines a 446 [gallo] 10 de henero en Burgos, vendió Carbajal, 1 marco, 6 uncias, 1 ochava a... 6 de febrero vendió Carbajal en Burgos, 7 uncias, 4 ochavas, 4 tomines, 4 granos a 445

13 de febrero en Burgos, vendió Juan Antonio 7 uncias, 6 ochavas, 1 tomín a 450

28 de febrero, vendió Juan Antonio en Burgos, 4 marcos, 5 uncias, 6 ochavas, 3 tomines a 453

15 de março y fue aluego, vendio Juan Antonio, en Burgos, 1 marco, y esto es lo de la funda al cual se le hizo cumplimiento a 453

Y fue 30 de diziembre, vendió Carbajal, en Burgos, al platero que ponía el sello, 4 uncias, 2 ochavas, 3 tomines a 448

24 de enero vendió Carbajal, en Burgos, 1 marco, 4 ochavas, 3 tomines a..8

3 de março dio Juan Antonio, por fechura del collar, 1 uncia, 2 tomines a 453, al cual platero se le debía 94 reales, por 47 aslavones que tiene a dos reales cada uno"

En este punto y hora Cristóbal Colón suspendió la cuenta itinerante de gastos personales, dejándonos con la miel en los labios.

Conocido el Memorial del oro en su integridad, poco nos resta que añadir sobre su presencia en la corte.

Sin embargo, por tratarse de una investigación "aurífera» parece conveniente puntualizar, como remate, las entregas de metal precioso público, cuando la calidad artística o la singularidad de las piezas lo recomiende.

El almirante acudía a Burgos para entrevistarse con los Reyes Católicos; informarles de los éxitos de la segunda expedición y promover los preparativos de la tercera empresa.

La entrevista con Isabel primero y con Fernando más tarde, sus generosos protectores, cada uno por separado, de acuerdo con la diversa entrada en la ciudad, y posteriormente de manera conjunta, agradeciéndole los servicios prestados, otorgándole mercedes y dictando un minucioso conjunto de órdenes para el apresto de la tercera exploración ${ }^{17}$.

Para deslumbrar a los monarcas abrió las arcas que conducía. El tesoro expuesto bastaria a llenar el espléndido museo: «coronas, carátulas, cintos, collares e otras muchas cosas entretexidas de algodón o de lo que era la alhaja. Traxo unas coronas con unas alas, en ellas unos ojos a los lados; e en especial traía una corona, que dezían que era del cacique Caonaboa, que era muy grande e alta, e tenía a los lados, estando tocado, unas alas como adargas e unos ojos

17 B Allesteros. Cristóbal Colón, tomo II, pp. 295-325

Higpantia, LIX/3, núm 203 (1999) $857-870$ 
de oro tamaños como taças de plata de medio marco cada uno, allí asentados como esmaltados por muy sotil e estraña manera, e allí el diablo figurado en aquella corona; e créese que así se les parecía y que eran idólatras e tenían al diablo por señon.

El cronista Andrés Bernáldez, nuestro informante, añade otros sustanciosos pormenores: "Traía un collar el dicho don Diego, hermano del Caonaboa, de oro, que le fazía el almirante poner cuando entrava por las ciudades o logares, fecho a eslabones de cadena, que pesava seiscientos castellanos; el cual yo vi e tomé en mis manos» ${ }^{18}$.

La solemne boda del príncipe don Juan con la princesa Margarita de Habsburgo, el 19 de marzo de 1497, conmocionó a toda la ciudad de Burgos, en el marco incomparable de la catedral. En lugar preferente el público pudo contemplar la hierática figura del descubridor del Nuevo Mundo luciendo sobre la dalmática el espléndido collar, recién labrado, para emular, en presencia, con los grandes de Castilla.

Las tecompensas vinieron a renglón seguido. Las más importantes fueron la confirmación, el 23 de abril de 1497, de los privilegios concedidos en la Vega de Granada y Barcelona con los títulos de almirante, virrey y gobernador de las Indias. A ello hay que añadir la facultad de fundar mayorazgo de todos sus bienes con los títulos anejos.

En idéntica fecha los soberanos de Castilla expiden una decena de provisiones y cédulas conducentes a la preparación de la tercera expedición al Nuevo Mundo ${ }^{19}$.

\section{JORNADA EN MEDINA DEL CAMPO. EL RETORNO}

La jornada regia en Burgos se alargó hasta el 9 de mayo de 1497 en que partieron para la villa de Medina del Campo a la que arribaron, con diversas paradas, el día $222^{20}$.

El almirante fue invitado a acompañarles para seguir perfilando la exploración y colonización de América.

Un nuevo episodio aurífero hay que destacar. Cristóbal Colón sorprendió al ilustre humanista milanés Pedro Mártir de Angleria al mostrarle la pieza más valiosa del tesoro que conducía. El sabio se expresa en estos términos: «Al explorar desde la fortaleza de la Concepción el interior de los montes de Cibao recibieron una masa de oro sin labrar, a manera de toba natural, cóncava, más grande que un puño, de veinte onzas de peso, que había encontrado un reyezuelo no en la ribera de ese río, sino en un montículo seco. A esta la ví, con mis propios ojos, en Medina del Campo, emporio de Castilla la Vieja, donde pasava

18 Memorias del reinado de los Reyes Católicos, pp. 333-334

19 BaLlesteros, pp. 302-306.

20 Rumeu DE Armas: Itinerario de los Reyes Católicos, pp. 234-235

Hìpania, LIX/3, núm. 203 (1999) 857-870 
Ia corte el invierno; y tomándola en mis manos, la sopesé y la manoseé admirado»s ${ }^{21}$.

La reina Isabel regaló el "grano de oro» a su fiel y leal servidor el almirante de las Indias. Sin embargo nuestro héroe lo retuvo en su poder tan sólo durante un año largo, pues en las vísperas de su tercera navegación a América decidió reintegrarlo a la soberana de Castilla.

El comisionado para la devolución fue su propio hijo Diego Colón. La carta escrita en Sevilla el 29 de abril de 1498 nos informa sobre el particular: «Ya te he escrito, con otra, que te enbiaré dos marcos de oro de nacimiento, de granos muy gordos; y así fago agora..., atado en un paño y asellado, para que lo ayas de dar a la Reina, nuestra señora, al tiempo que virdes que mejor venga, con acoerdo de Jerónimo [de Agüero] y del tesoirero Villacurta...; y a mi ver será bien cuan acaba de comens.

Insistiendo en el valor intrínseco de la presea, prosigue: «El es tal gioya que antes he sofrido mil necesidades que venderlo ni fondirle, para servir con él a la Reina, nuestra señora, pues Su Alteza me había fecho merced dello; mas vi que era cargo de consçiençia a desfazele, salvo bolvéselo, porque vea los milagros de Nuestro Señor y le haga ver a quien Su Alteza aplogiere» ${ }^{22}$.

Habrá sorprendido al lector la recomendación del almirante a su hijo de devolver la joya a la soberana de Castilla "cuan acaba de comen. La explicación es sencilla: si el obsequio había sido público, la devolución debería tener el mismo carácter y resonancia.

Se impone situarnos de nuevo en Medina del Campo. De alli partió para Sevilla en el verano de 1497 , sin que se pueda precisar la fecha exacta ${ }^{23}$.

\footnotetext{
21 Décadas de Orbe Novo. Edición y traducción de Juan Gil, Alianza Editorial, Madrid 1984, p. 80.

22 DuQuesa De Berwick y DE Alba: Autógrafos de Cristóbal Colón y Relaciones de Uliramar, Madrid 1902, p. 11

23 RUMEU DE ARmas, Antonio: Itinerario de los Reyes Católicos, pp. 235-236
}

IHiptunia, LIX/3, núm. 203 (1999) 857-870 


\section{APENDICE}

\section{Transcripción}

\section{Memorial del oro que se ba vendido en Castilla, fasta el mes de}

A XX de julio, en Sebilla, vendió Cristóbal de Torres 2 marcos, 7 onças, 4 ochavas de oro a 453 el peso del castellano.

A 12 d'agosto, en Valladolid, vendió Carbajal 2 marcos, 6 uncias, 4 ochavas, 3 tomines, y to dio casi todo en ropa que se compró.

11 de setiembre, en Arcos, vendió Carbajal 6 uncjas, 2 ochavas, 1 tomín, 3 granos, vendiólo a $* * *$

19 de setiembre, en Burgos, se pesó 4 uncias, 7 ochavas, 3 tomines a 445

[grano] a 7 de otubre, en Burgos vendió Carbajal 7 uncias, 4 ochavas, 5 tomines, 3 granos

25 d'otubre, en Burgos, vendió Carbajal 7 uncias, 4 ochavas, 1 tomin, 10 granos a 445

12 de noviembre, en Burgos, vendió Carbajal 1 marco, 6 uncias, 7 ochavas menos 14 granos, a 448

17 de deziembre, vendió en Burgos Carbajal, 7 uncias, 5 ochavas, 3 tomines a 446

[gallo] 10 de henero en Burgos, vendió Carbajal, 1 marco, 6 uncias, 1 ochava [s] a***

6 de febrero vendió Carbajal en Burgos, 7 uncias, 4 ochavas, 4 tomines, 4 granos a 445

13 de febrero en Burgos, vendió Juan Antonio 7 uncias, 6 ochavas, 1 tomín a 450

28 de febrero, vendió Juan Antonio en Burgos, 4 marcos, 5 uncias, 6 ochavas, 3 tomines a 453

15 de março y fue aluego, vendio Juan Antonio, en Burgos 1 marco, y esto es lo de la funda al cual se le hizo complimiento a $\mathbf{4 5 3}$

Y fue 30 de deziembre, vendió Carbajal, en Burgos, al platero que ponía el sello, 4 uncias, 2 ochavas, 3 tomines a 448

24 de enero vendió Carbajal, en Burgos, 1 marco , 4 oclsavas, 3 tomines a ** 8

3 de março dio Juan Antonio, por fechura del collar, 1 uncia, 2 tomines a 453, al cual platero se le debía 94 reales, por 47 aslavones que tiene a dos reales cada uno.

\section{en el ángulo inferior izquierdo}

ducentos treinta e cuatro docados que me dió don Diego a... 


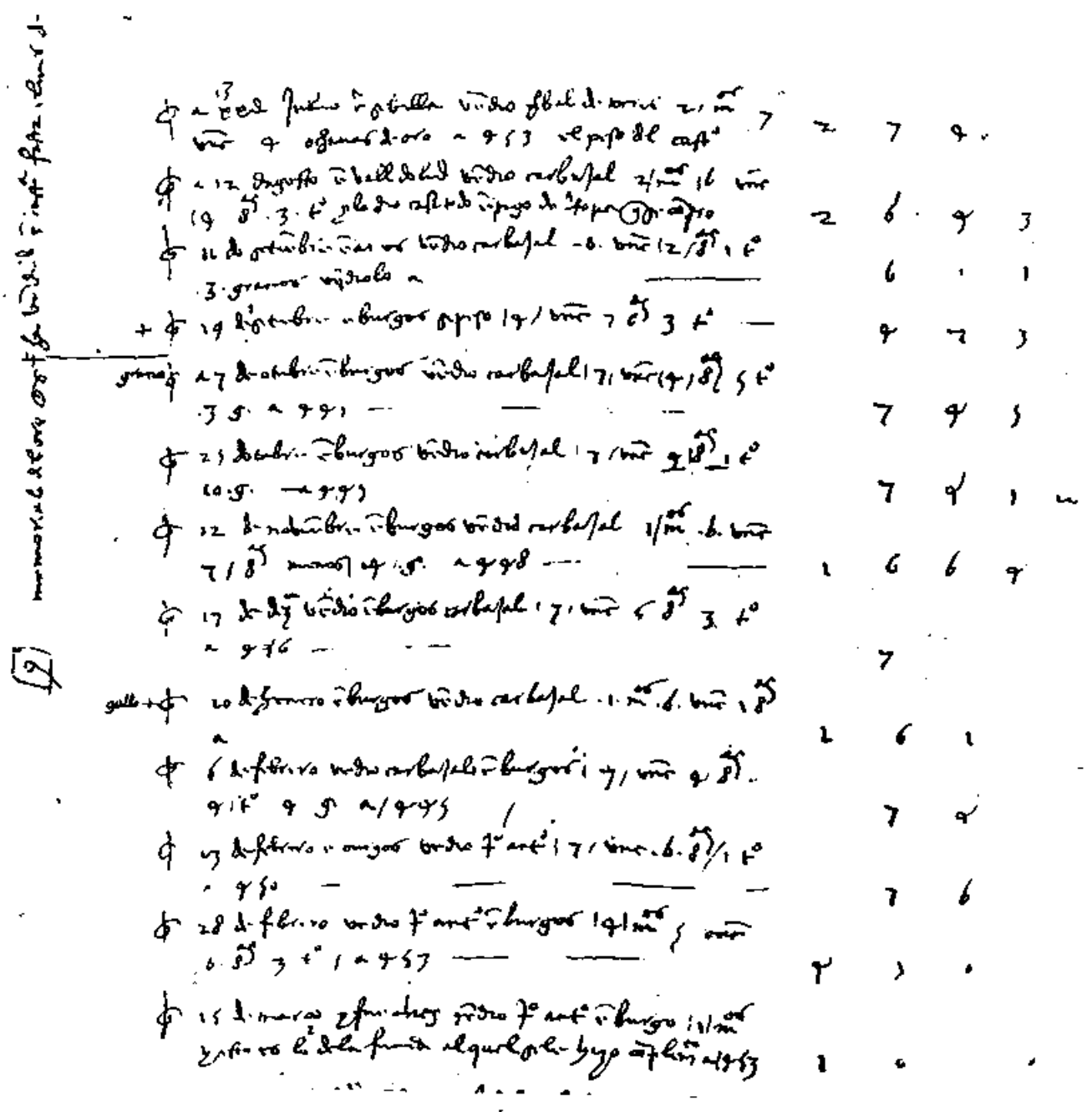




\section{APENDICE II}

\section{TABLA DE EQUIVAIENCIAS}

$\begin{array}{lr}1 \text { marco }= & 230 \text { gramos/oro. } \\ 1 \text { onza (uncia) } & 28,75 \text { gramos/oro. } \\ 1 \text { ochava = } & 3,59 \text { gramos/oro. } \\ 1 \text { tomin = } & 0,59 \text { gramos/ora. } \\ 1 \text { grano = } & 0,049 \text { gramos/oro. } \\ 1 \text { castellano de oro = 4,6 gramos /oro. }\end{array}$

NƠ'A: La cotización del castellano en maravedies cambiaba como consta en cl texto. Lin caso que no se haga constar la equivalencia entre el castellano y el maravedí, se ha calculado en función de la cotización oficial media de las cecas castcllanas, que era de 1 castellano $=450$ maravedies.

1 (Sevilla, 20 de julio)

$\begin{array}{lc}2 \text { marcos } \times 230= & 460 \\ 7 \text { onzas } \times 28,75= & 201,25 \\ 4 \text { ochavas } \times 3,59= & 14,36 \\ \text { TOTAL..................... } & 676,61 \text { qrs. }\end{array}$

$675,61: 4,6$ grs. por castellano $=146,87$ castellanos

$146,87 \times 453$ maravedies $=66.532,11$ maravedies

2 (Valladolid, 12 de agosto)

$2 \operatorname{marcos} \times 230=$

6 uncias $\times 28,75=$

4 ochavas $\times 3,59=$

3 tomines $\times 0,50=$

TOTAL.

460
172,5
14,36
1,77
648,63 grs.

$648,63: 4,6=141$ castellanos

$141 \times 450=63.450$ maravedíes.

3 (Arcos, 11 de septiembre)

6 uncias $\times 28,75=$

2 ochavas $\times 3,59=$

1 tomín $\times 0,59=$

3 granos $\times 0,049=$

TOTAL

$180,417: 4,6=39,22$ castellanos

$39,22 \times 450=17.649$ maravedies

4 (Burgos, 19 de septiembre)

4 uncias $\times 28,75=$

7 ochavas $\times 3,59=$

3 tomines $\times 0,59=$

TOTAL.

$141,9: 4,6=30,84$ castellanos.

$30,84 \times 450=13.878$ maravedies.

5 (Burqos, 7 de octubre)

7 uncias $\times 28,75=$

4 ochavas $\times 3,59=$

5 tomines $\times 0,59=$

3 granos $\times 0,049=$

TOTAI

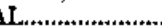

Hispania, LIX/3, núm. 203 (1999) 857-870 


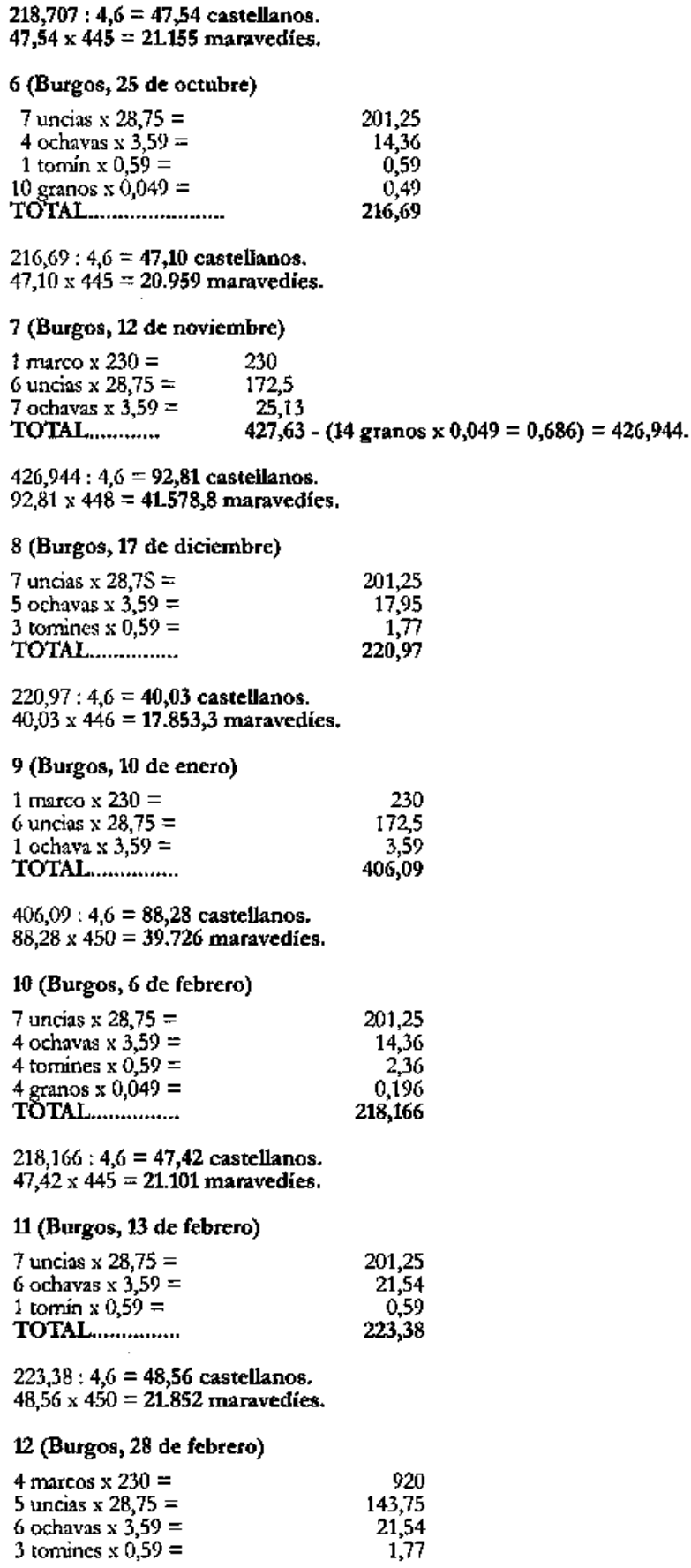

$218,166: 4,6=47,42$ castellanos. $47,42 \times 445=21.101$ maravedies.

11 (Burgos, 13 de febrero)

7 uncias $\times 28,75=$

6 ochavas $\times 3,59=$

1 tomin $x 0,59=$

$223,38: 4,6=48,56$ castellanos $48,56 \times 450=21.852$ maravedies.

12 (Burgos, 28 de febreso)

$4 \operatorname{marcos} \times 230=$

5 uncias $\times 28,75=$

6 ochavas $\times 3,59=$

3 tomines $\times 0,59=$ 
870

ANTONIO RUMEU DE ARMAS

TOTAL

$1.087,06$

$1.087,06: 4,6=236,31$ castellanos.

$236,31 \times 453=107,048,43$ maravedies.

13 (Burgos, 15 de marzo)

1 marco $230=$

230

TOTAL..............

230

$230: 4,6=50$ castellanos.

$50 \times 453$ - 22,650 maravedies.

14 (Burgos, 30 de diciembre)

4 uncias $\times 28,75=$

2 ochavas $\times 3,59=$

3 tomines $\times 0,59=$

TOTAL

$123,95: 4,6=26,94$ castellanos.

$26,94 \times 44 B=12.069,12$ maravedies.

15 (Burgos, 24 de enero)

1 marco $\times 230=$

4 ochavas $\times 3,59=$

3 tomines $\times 0,59=$

TOTAL.

$246,13: 4,6=53,50$ castellanos

$53,50 \times 448=23.968$ maravedies.

16 (s.l., 3 de marzo)

1 uncia $\times 28,75=$

2 tomines $\times 0,59=$

TOTAI

$29,93: 4,6=6,50$ castellanos.

$6,50 \times 453=2,944,5$ maravedies.

Hitpatuitu, LIX/3, núm. 203 (1999) 857-870 